\title{
Overexpression of copper/zinc superoxide dismutase in the median preoptic nucleus improves cardiac function after myocardial infarction in the rat
}

\author{
John P. Collister ${ }^{1}$, Cristina Hartnett ${ }^{1}$, Tim Mayerhofer ${ }^{1}$, David Nahey ${ }^{1}$, Christopher \\ Stauthammer ${ }^{2}$, Maxie Krüger ${ }^{2}$, Anthony Tobias $^{2}$, M. Gerard O'Sullivan ${ }^{3}$, Josh Parker ${ }^{3}$, Jun \\ Tian $^{4}$, Adam J. Case ${ }^{4}$, and Matthew C. Zimmerman ${ }^{4}$ \\ ${ }^{1}$ Department of Veterinary and Biomedical Sciences, College of Veterinary Medicine University of \\ Minnesota St. Paul, MN \\ ${ }^{2}$ Department of Veterinary Clinical Sciences, College of Veterinary Medicine University of \\ Minnesota St. Paul, MN \\ ${ }^{3}$ Department of Veterinary Population Medicine College of Veterinary Medicine University of \\ Minnesota St. Paul, MN \\ ${ }^{4}$ Department of Cellular and Integrative Physiology University of Nebraska Medical Center \\ Omaha, NE
}

\section{Abstract}

Previous reports indicate that overexpression of copper/zinc superoxide dismutase (CuZnSOD), an intracellular superoxide $\left(\mathrm{O}_{2}{ }^{-}\right.$) scavenging enzyme, in the brain subfornical organ improves cardiac function in a mouse model of heart failure (HF). A downstream hypothalamic site, the $\mathrm{MnPO}$, may act as a relay center for $\mathrm{O}_{2}{ }^{--}$to serve as a mediator in the pathophysiology of HF. To test the hypothesis that elevated $\mathrm{O}_{2}{ }^{--}$in the $\mathrm{MnPO}$ contributes to the pathophysiology of $\mathrm{HF}$ and decreased cardiac function, we injected adenovirus encoding CuZnSOD (AdCuZnSOD, n=7) or control empty adenovirus vector (AdEmpty, $\mathrm{n}=7$ ) into the $\mathrm{MnPO}$ of normal rats. Subsequently, rats were subjected to coronary artery ligation to create a myocardial infarct (MI) of the left ventricle. Cardiac function was monitored via echocardiography. Upon completion, rat brains were examined for CuZnSOD expression in MnPO via immunofluorescence and histopathological analyses of cardiac infarct size were conducted. Baseline (EF) ejection fractions (\%) of AdCuZnSOD and AdEmpty rats were $73 \pm 1$ and $71 \pm 1$, respectively. Two weeks after MI, EF was significantly decreased in both groups of rats (AdCuZnSOD: 51 \pm 3 , AdEmpty: 46 \pm 1 ). In contrast by 4 weeks post MI, EF had improved to $64 \pm 2$ in AdCuZnSOD rats yet was only $52 \pm 1$ in AdEmpty rats, and this was accompanied by lower plasma norepinephrine levels in AdCuZnSOD rats $(0.49 \pm 0.19 \mathrm{ng} / \mathrm{ml})$ compared to AdEmpty rats $(1.20 \pm 0.32 \mathrm{ng} / \mathrm{ml})$. In conclusion, despite decreases in EF early after MI, overexpression of CuZnSOD in the MnPO was related to an improvement in left ventricular function and concomitant decreased plasma norepinephrine levels 4 weeks post MI.

Corresponding Author: John P. Collister, University of Minnesota, Department of Veterinary Biosciences, University of Minnesota, colli066@umn.edu Phone: 612-626-1206. 


\section{Keywords}

Myocardial infarction; Heart failure; median preoptic nucleus; superoxide dismutase; norepinephrine

\section{INTRODUCTION}

Myocardial ischemia and infarction (MI) is the primary cause of systolic heart failure (HF) (1). For years it has been well accepted that in many forms of clinical and experimentally induced HF, activation of the renin angiotensin system (RAS) and sympathetic nervous system (SNS) occurs (2-4). In HF, sympathetic activity increases such that it follows the decline in cardiac performance $(4,5)$ and may contribute to the progression of heart failure (6). Abnormalities in the central nervous system (CNS) resulting in excessive sympathetic drive are strongly implicated in the progression to heart failure after events such as myocardial infarction (7-9). In fact, increased levels of plasma norepinephrine are linked to poor prognosis (10) and sympathoexcitation associated with heart failure induced cardiomyocyte death has been established (11-13). While recent studies have implicated the CNS in this regard, little is known of the actual pathways leading to increased sympathetic activity.

Certainly there are links between the increased RAS and SNS, however little is known of the initial signaling pathways during post MI-HF leading to this increased central angiotensinergic activity and sympathoexcitation. The peripheral RAS is rapidly activated post MI, likely by immediate drops in blood pressure, and is mildly elevated in the subsequent weeks as heart failure develops (14). This increase in circulating angiotensin II (AngII) could certainly be involved as well with central pathways mediating increased angiotensinergic and sympathetic nervous activity. The central circumventricular organs (CVO) lack the normal blood brain barrier and are well known to be responsive to changes in circulating AngII $(15,16)$. The subfornical organ (SFO) and organum vasculosum of the lamina terminalis (OVLT) are two forebrain CVOs with known actions mediating effects of circulating AngII (17).

Indeed, the need to study these specific areas of the forebrain in regard to the homeostatic changes and pathophysiology of MI-induced HF is enhanced by studies examining the anteroventral portion of the 3rd ventricle (AV3V) in models of HF as well. The AV3V itself contains the ventral MnPO, the OVLT and descending fibers of the SFO (18). Therefore a lesion of this area disrupts a huge portion of the forebrain lamina terminalis and as such has been examined in the MI-HF model. Lesion of the AV3V blocked some of the initial sodium and water balance changes seen after MI, caused an attenuation in sodium appetite, and prevented renal sodium and water retention $(9,19,20)$. Additionally and surprisingly, it completely prevented the rise in plasma renin activity seen in normal rats after MI $(9,19,20)$. It also caused a reduction in HR, MAP and renal sympathetic nerve activity. While all of these results were notable to ameliorate the seemingly detrimental rise in SNS and RAS activity, an increased mortality was seen in AV3V lesioned rats by 3 weeks post MI 
$(9,19,20)$. These results highlight the importance of further investigation into which specific areas of the AV3V are involved in this important regulation post MI.

Regarding potential neuronal cellular signaling mechanisms involved in mediating the sympathoexcitation of post MI-HF, there are a number of possibilities. Redox signaling has recently been given much attention as a potential mechanism in the pathophysiology of HF. Elevated levels of reactive oxygen species (ROS) have been shown in plasma of HF patients and are known to impair cardiac performance in many experimental models (21). Previous reports have shown that adenoviral-mediated overexpression of copper/zinc superoxide dismutase (CuZnSOD) and increased scavenging of superoxide $\left(\mathrm{O}_{2}{ }^{--}\right)$in the SFO diminishes post MI induced sympathoexcitation (22). More importantly, the increased scavenging of $\mathrm{O}_{2}^{--}$in the SFO resulted in improved myocardial function, decreased cardiac myocyte apoptosis, and increased survival rates (23). Similar observations have been made in animals overexpressing CuZnSOD in the paraventricular nucleus (PVN) (24), a cardiovascular control brain region that receives input from the SFO and contributes to cardiovascular function via modulation of sympathetic output and vasopressin release. As a potential relay station between the SFO and PVN, the MnPO is a likely candidate where redox signaling may be an important mechanism in the central pathophysiology post MI. As such, using adenoviral mediated overexpression of CuZnSOD (AdCuZnSOD), we tested the hypothesis that elevated $\mathrm{O}_{2}{ }^{-}$in the MnPO plays a role in the pathophysiology of heart failure and decreased cardiac function post MI in the rat. Our results demonstrate that despite decreases in ejection fraction early after MI, overexpression of CuZnSOD in the $\mathrm{MnPO}$ was related to an improvement in left ventricular function at both 4 and 6 weeks post MI. Improved ventricular function in AdCuZnSOD treated rats was also related to significantly less circulating norepinephrine. These results support the hypothesis that elevated $\mathrm{O}_{2}{ }^{--}$in the MnPO contributes to the pathophysiology and decreased left ventricular function post MI.

\section{RESULTS}

\section{Effect of CuZnSOD overexpression in the MnPO on cardiac function after MI}

Figure 1A shows representative M-mode echocardiograms from a rat at baseline and 2 weeks post-MI. At baseline, normal left ventricular chamber dimensions and normal systolic function are observed as indicated by a shortening fraction (SF) of $42 \%$ and ejection fraction (EF) of $81 \%$. In contrast, increased left ventricular chamber dimensions and markedly depressed left ventricular systolic function as indicated by a SF of $24 \%$ and an EF of $41 \%$ is observed at 2 weeks post-MI. Figure 1B demonstrates that average baseline EF (\%) were not different between AdCuZnSOD- and AdEmpty-injected rats before myocardial infarction (AdCuZnSOD; 73 \pm 1 , AdEmpty; 71 \pm 1 ). Furthermore, 2 weeks post-MI both groups of rats showed a similar and marked decrease in EF that was significantly decreased from baseline (Figure 1B). However, although the EF of either group did not return to normal levels compared to baseline, at 4 and 6 weeks post-MI, EF did improve in AdCuZnSOD-injected rats (Figure 1B) such that it was statistically higher than that observed in AdEmpty-treated rats (4 weeks post MI: AdCuZnSOD; 64 \pm 2 , AdEmpty; 52 \pm 1 ). A summary of ejection fraction data from both groups of rats over time (before and after MI) is provided in Table 1. 


\section{Effect of CuZnSOD overexpression in the MnPO on plasma norepinephrine levels after MI}

Plasma norepinephrine levels from AdCuZnSOD and AdEmpty rats at 2 and 4 weeks post

MI are shown in Figure 2. There was no difference in norepinephrine levels between groups at 2 weeks post MI (AdCuZnSOD; $0.59 \pm 0.21 \mathrm{ng} / \mathrm{ml}$, AdEmpty; $0.77 \pm 0.35 \mathrm{ng} / \mathrm{ml})$. In contrast, AdEmpty treated rats had elevated norepinephrine levels $(1.20 \pm 0.32 \mathrm{ng} / \mathrm{ml})$ compared to $\mathrm{AdCuZnSOD}$ treated rats $(0.49 \pm 0.19 \mathrm{ng} / \mathrm{ml})$ at 4 weeks post MI.

\section{Infarct measurements and Immunofluorescence of AdCuZnSOD}

Representative tissue sections from a MI-sham rat and a MI rat 6 weeks post-MI are shown in Figure 3. Using digital imaging software, the area of infarct in the representative MI rat section was measured at $10.341 \mathrm{~mm}^{2}$ compared to $39.987 \mathrm{~mm}^{2}$ remaining ventricular free wall and interventricular septum. The lesion shown is a representative and typical infarcted heart from rats used in this study. Infarcted hearts from both groups were analyzed and shown to be similar in location and size. The average LV infarct sizes were calculated for each group and shown to be similar in size. The average infarct size (\%) in AdCuZnSOD rats was $20 \pm 1(n=7)$ compared to $17 \pm 1(n=7)$ in AdEmpty rats. There was no statistical difference of mean infarct size between groups.

To verify adenoviral-mediated CuZnSOD overexpression in the MnPO, immunofluorescence and confocal microscopy were performed on brains of all rats following completion of the protocol. Figure 4A is a schematic of a coronal forebrain section showing the location of the $\mathrm{MnPO}$ (highlighted in green). Figure 4B shows representative confocal images of coronal forebrain sections of immunofluorescent staining for CuZnSOD protein (green fluorescence) in the dorsal MnPO approximately 6 weeks after direct injection of AdCuZnSOD or AdEmpty into the MnPO. All AdCuZnSOD rats included in the final analyses were confirmed to have robust expression of CuZnSOD in and confined to the MnPO relative to the minimal endogenous CuZnSOD detected in the MnPO of AdEmpty rats.

\section{DISCUSSION}

Post myocardial infarction induced heart failure is characterized by increased neurohumoral activity that leads to sympathoexcitation that is eventually detrimental to the myocardial workload and leads to deterioration in ventricular function $(27,28)$. The overall sympathoexcitation observed during heart failure is thought to be driven via the CNS $(7,9,27)$. While the entire milieu of peripheral and central signaling mechanisms involved in this response is vast, much attention has recently been focused on the role of ROS, particularly $\mathrm{O}_{2}{ }^{-}$, in the brain in cardiovascular disease states such as heart failure. Scavenging $\mathrm{O}_{2}{ }^{--}$in the forebrain SFO of MI-induced heart failure mice has been shown to improve myocardial function (23). The SFO is one of the circumventricular organs of the CNS, and as such is capable as functioning as a sensor of circulating hormones and causing downstream neuronal activation of hypothalamic areas such as the PVN, which in turn has been linked to sympathoexcitation (8). The MnPO is one of the downstream integrative nuclei of the SFO which projects to the PVN as well and as such, in the present study we investigated the role of $\mathrm{O}_{2}{ }^{-}$in this regulatory nucleus in the deterioration of ventricular function in the post MI rat. Using adenoviral vectors capable of causing overexpression of 
CuZnSOD injected directly into the MnPO we were able to show that AdCuZnSOD treated rats had less impairment in left ventricular function 4 and 6 weeks post myocardial infarction. Interestingly, the relative improvement in cardiac function in AdCuZnSOD treated rats at 4 weeks post MI was accompanied by lower plasma norepinephrine levels as compared to AdEmpty-treated rats, which had sustained lower ejection fractions. Our results demonstrate that increased levels of $\mathrm{O}_{2}{ }^{--}$in the $\mathrm{MnPO}$ as well as increased overall sympathetic activity demonstrated by elevated norepinephrine levels, contribute to the decline in myocardial function after MI in the rat.

While many hormonal and inflammatory pathways are activated during heart failure, the peripheral RAS, and a concomitant rise in circulating AngII, as well as central RAS activity are likely candidates driving the central activation of the sympathetic nervous system during heart failure. Certainly there are links between the increased RAS and SNS and it is readily apparent that central angiotensinergic pathways are involved with HF associated sympathoexcitation. In a HF model induced in dogs, AngII levels have been shown to be increased in the cerebrospinal fluid after congestive failure ensues (29). Additionally, central $\mathrm{AT}_{1}$ receptor expression is increased in known sympathetic centers such as the PVN and rostral ventral lateral medulla (RVLM) after induction of $\operatorname{HF}(30,31)$. The PVN has also been shown to have increased neuronal activity after MI induced HF in the rat, which was reduced by prior intracarotid treatment with either the $\mathrm{AT}_{1}$ antagonist, losartan or the angiotensin converting enzyme (ACE) inhibitor, captopril (32). Furthermore, both the $\mathrm{AT}_{1}$ receptor antagonist, losartan, or $\mathrm{AT}_{1}$ receptor antisense infused intracerebroventricularly reduced the increase in renal sympathetic nerve activity in post myocardial infarction (MI) induced heart failure in the rat (33-35). Lastly, transgenic rats expressing antisense RNA against angiotensinogen mRNA specifically in the brain demonstrated less impaired cardiac ventricular function and remodeling post MI compared to normal rats with similar MI (36,37). These data provide evidence for central RAS involvement in sympathoexcitation and progressive cardiac decline in function during post MI induced heart failure.

Elevated levels of ROS, particularly $\mathrm{O}_{2}{ }^{--}$, and enhanced redox signaling in the CNS has recently been given much attention as a potential mechanism in the pathophysiology of HF. It was first demonstrated that injection of SOD into the brainstem attenuated increased sympathetic tone in pigs receiving chronic nitrate treatment (38). Additional studies demonstrated that NADPH oxidase, an intracellular source of $\mathrm{O}_{2}{ }^{--}$, in the RVLM plays a role in the sympathoexcitation in a rabbit model of $\mathrm{HF}$ (30), and that the elevated $\mathrm{O}_{2}{ }^{--}$levels in the RVLM are diminished by prior treatment with ICV losartan (31). Furthermore, overexpression of CuZnSOD via in the brain attenuated the increased renal sympathetic nerve activity seen during HF (22). Additionally, CuZnSOD overexpression in the SFO and increase scavenging of $\mathrm{O}_{2}{ }^{--}$in this cardiovascular control brain nuclei was accompanied by improved myocardial function, decreased cardiac myocyte apoptosis, as well as increased survival rates in MI rats (23). Similar results have been reported in animals with adenovirusmediated overexpression of CuZnSOD in the PVN (24). As a potential relay station between the SFO and PVN, the MnPO is a likely candidate where elevated levels of $\mathrm{O}_{2}{ }^{--}$may be an important mechanism in the central pathophysiology post MI. Our current results showed that both AdCuZnSOD and control (AdEmpty) rats had similar impairment in left ventricular function as shown by significant decreased EF 2 weeks post MI. However, by 4 
and 6 weeks post MI, rats treated with AdCuZnSOD had improved EF that was significantly higher compared to AdEmpty-treated control rats. This observation was accompanied by a significant rise in plasma norepinephrine levels in AdEmpty rats from 2 weeks to 4 weeks post-MI that was not observed in AdCuZnSOD rats. While EF did not return to normal in AdCuZnSOD rats, these results demonstrate that specific treatment of AdCuZnSOD in the MnPO of MI rats improves cardiac function chronically in MI rats and support the notion that the chronic impairment of left ventricular function in AdEmpty rats is sympathetically mediated.

While infarct sizes were similar in both groups of rats, we did not investigate any cellular changes in the myocardium that could account for the observed changes in myocardial function in this study. Previous studies have suggested that following MI, cellular apoptosis plays a significant role in the deterioration of the myocardium and contributes to left ventricular dysfunction $(39,40)$. Similar to the present study, the previous experiments of Lindley, et. al. demonstrated similar infarct sizes in centrally administered AdCuZnSOD mice compared with control mice (23). While using indices of apoptosis, they reported less left ventricular apoptosis in mice with CuZnSOD overexpression in the SFO, suggesting that overexpressing SOD in the SFO is protective and preserves some of the integrity of the infracted myocardium, despite the observation that the infarct sizes themselves were similar in both groups of rats (23). Along this line of reasoning, it has been reported that sympathoexcitation can cause increased myocardial apoptosis $(12,13,28)$, and central scavenging of $\mathrm{O}_{2}{ }^{--}$reduces sympathetic drive (22). Indeed, our current results also suggest a lower level of sympathetic activity in AdCuZnSOD rats beginning at 4 weeks post MI compared to control rats that maintained a lower level of ventricular function. Taken together, it is plausible that central administration of AdCuZnSOD in the MnPO, may lessen cardiac dysfunction by leading to a decrease in the myocardial apoptosis in the post MI rat.

Our results support and extend findings of others in that central administration of AdCuZnSOD, specifically in the MnPO, ameliorates part of the left ventricular dysfunction observed chronically after MI in the rat. While others have shown similar results in the SFO and PVN, taken together these results demonstrate the importance of future investigations of novel antioxidant therapies aimed at the SFO-MnPO-PVN pathway and the role of superoxide in the deleterious sympathoexcitatory effects in the post MI heart.

\section{MATERIALS AND METHODS}

All methods were approved by the Institutional Animal Care and Use Committee and conducted in accordance with institutional and National Institutes of Health guidelines. Male Sprague-Dawley rats (Charles River Laboratory, Wilmington, MA, USA) weighing 250-275 $\mathrm{g}$ were used for experiments and housed in an animal housing facility with a $12 \mathrm{~h}-12 \mathrm{~h}$ lightdark cycle.

\section{Surgical Procedures}

Rats were randomly divided into 2 groups (AdCuZnSOD; $n=7$ or AdEmpty; $\mathrm{n}=7$ ), anesthetized with pentobarbital (65 mg/kg IP) and atropine $(0.2 \mathrm{mg} / \mathrm{kg} \mathrm{IP})$, and placed in a stereotaxic apparatus (model no. 900; David Kopf Instruments; Tujunga, CA, USA). A 
dorsal midline incision was made in the skin of the skull. Bregma and lambda were exposed, repositioned to be on the same horizontal level, and a $2.0 \mathrm{~mm}$ hole was drilled into the skull. Adenoviral vectors (Viraquest, North Liberty, IA) encoding human copper/zinc cytoplasmic superoxide dismutase (AdCuZnSOD) and control vector (AdEmpty) were injected into the $\mathrm{MnPO}$ of rats, respectively. Titers of viral vectors were pair matched at $10^{9} \mathrm{pfu} / \mathrm{ml}$. A Hamilton syringe was lowered through the midline hole in the skull to the following 2 coordinates (mm caudal and ventral relative to bregma): $-0.35,-7.2$ and $-0.4,-.1$, allowing placement of the injector near both the dorsal and ventral aspects of the MnPO. $100 \mathrm{nl}$ of virus was injected at both locations in the $\mathrm{MnPO}$ over a period of 5-10 minutes. The hole in the skull was repaired with bone wax and the skin closed with 3-0 $(0.2 \mathrm{~mm})$ silk suture.

After surgery, rats were given an injection of the antibiotic gentamicin (2.5 mg, I.M.) and the analgesic butorphanol tartrate $(0.075 \mathrm{mg}$, S.C. $)$.

One week later, a femoral venous catheter was placed and a myocardial infarct (MI) was induced in all rats via ligation of the left anterior descending (LAD) coronary artery as previously described $(20,21)$. Rats were anesthetized with isoflurane, intubated and mechanically ventilated at of rate of 55 breaths/min and tidal volume of $2.5 \mathrm{ml}$. Briefly, a left thoracotomy was performed and the pericardium opened. The LAD was ligated between the pulmonary outflow tract and left atrium with 6.0 silk suture. The chest incision was closed with 3-0 silk suture while negative thoracic pressure was restored via a 23 gauge butterfly catheter attached to a three-way stopcock and 3cc syringe. Sham operated rats were prepared similarly but without LAD ligation. Lidocaine $(2 \mathrm{mg})$ was given IM upon coronary ligation and 4 hours post-operatively. A catheter was then implanted in the femoral vein for whole blood sampling. The catheter was tunneled subcutaneously, exteriorized and capped between the scapula through a dacron mesh button tether (Harvard Apparatus Inc., Holliston, MA, USA), and the mesh sutured to the interscapular muscle. Antibiotics and analgesics were provided as above.

\section{Experimental Procedures}

Transthoracic echocardiography was performed on all rats 0,2, 4 and 6 weeks post MI. Animals were briefly anesthetized with isoflurane and placed in right lateral recumbency. The chest was shaved and pre-warmed acoustic coupling gel applied. Images were obtained with a $12-5 \mathrm{mHz}$ transducer. Short axis images were acquired at the level of the papillary muscle tips parallel to the mitral valve plane. The following measurements were made: left ventricular end diastolic and systolic diameters (LVEDD and LVESD, respectively). These measurements were then used to compute LVEDV and LVESV using the Teichholz method (25), and ejection fractions were calculated as ((LVEDV minus LVESV)/LVEDV * 100).

Prior to echocardiography, blood was obtained from all rats 2 and 4 weeks post MI. Briefly, one $\mathrm{ml}$ of whole blood was obtained via the femoral catheter and placed into a chilled $1 \mathrm{ml}$ syringe containing $1 \mathrm{mg}$ EDTA in $20 \mu \mathrm{l}$. Whole blood was centrifuged and plasma collected and stored at $-80^{\circ} \mathrm{C}$ for later ELISA analysis for norepinephrine (Norepinephrine ELISA Fast Track \#BAE-6200, Rocky Mountain Diagnostics, Inc., Colorado Springs, CO.)

At the end of the protocol, all rats were deeply anesthetized and perfused with heparinized saline $(20 \mathrm{U} / \mathrm{ml} ; 150 \mathrm{ml})$ followed by $4.0 \%$ paraformaldehyde via the aorta. Brains were 
removed, post fixed overnight and transferred to $20 \%$ sucrose. Brains were processed for immunofluorescent detection of CuZnSOD protein as previously described (26). Briefly, brain sections were incubated with human CuZnSOD antibody (sheep anti-human IgG diluted 1:500 in $2 \%$ NHS and $0.3 \%$ Triton overnight at $4^{\circ} \mathrm{C}$ ), washed, and further incubated with donkey anti-sheep FITC conjugated secondary antibody (diluted 1:200). Sections were washed, mounted on slides and imaged with confocal microscopy.

After perfusion, hearts were removed, placed in $4.0 \%$ paraformaldehyde overnight and midLV sections were mounted onto glass slides. Alternating samples were counterstained with hematoxylin and eosin, or trichrome stain. Digital images of the sections were acquired, LV fibrosis quantified and used to determine the infarct size.

\section{Statistical Analysis}

The results are reported as means \pm SE. A Student $t$-test or one-way ANOVA with repeated measures combined with a Student-Newman-Keuls test was used for comparisons.

Differences were considered significant at $\mathrm{p}<0.05$.

\section{ACKNOWLEDGMENTS}

This study was supported, in part, by the University of Minnesota College of Veterinary Medicine Signature Program in Comparative Medicine and the National Heart, Lung, and Blood Institute at the National Institutes of Health (R01HL103942 to M.C.Z). We thank Janice A. Taylor and James R. Talaska of the Confocal Laser Scanning Microscope Core Facility at the University of Nebraska Medical Center for providing assistance with confocal microscopy and the Nebraska Research Initiative and the Eppley Cancer Center for their support of the Core Facility. We thank Mr. Michael O'Keefe for technical assistance and construction of equipment used during the echocardiography of the experimental rodents.

\section{REFERENCES}

1. Rosamond W, Flegal K, Furie K, et al. American Heart Association Statistics Committee and Stroke Statistics Subcommittee. Heart Disease and Stroke Statistics 2008 Update A Report from the American Heart Association Statistics Committee and Stroke Statistics Subcommittee [online]. Circulation. 2008; 117:e25-e146. [PubMed: 18086926]

2. Ferguson DW, Berg WJ, Sanders JS. Clinical and hemodynamic correlates of sympathetic nerve activity in normal humans and patients with heart failure: evidence from direct microneurographic recordings. J. Am. Coll. Cardiol. 1990; 16(5):1125-1134. [PubMed: 2229759]

3. Liu JL, Zucker IH. Regulation of sympathetic nerve activity in heart failure: a role for nitric oxide and angiotensin II. Circ Res. Mar 5; 1999 84(4):417-23. [PubMed: 10066676]

4. Leimbach WN, Wallin BG, Victor GR, Aylward PE, Sundlof G, Mark AL. Direct evidence from intraneural recordings for increased central sympathetic outflow in patients with heart failure. Circulation. 1986; 73:913-919. [PubMed: 3698236]

5. Meredith IT, Eisenhofer G, Lambert GW, Dewer EM, Jennings GL, Esler MD. Cardiac sympathetic nervous activity in congestive heart failure: evidence for increased neuronal epinephrine release and preserved neuronal uptake. Circulation. 1993; 88:136-145. [PubMed: 8391399]

6. Packer M. Neurohumoral interactions and adaptations in congestive heart failure. Circulation. 1988; 77:721-730. [PubMed: 3280156]

7. Zucker IH. Novel mechanisms of sympathetic regulation in chronic heart failure. Hypertension. 2006; 48:1005-1011. [PubMed: 17015773]

8. Leenen FH. Brain mechanisms contributing to sympathetic hyperactivity and heart failure. Circ. Res. 2007; 101:221-223. [PubMed: 17673679]

9. Felder RB, Francis J, Zhang ZH, Wei SG, Weiss RM, Johnson AK. Heart failure and the brain: new perspectives. Am. J. Physiol. 2003; 284:R259-R276. 
10. Cohn JN, Levine TB, Olivari MT, Garberg V, Lura D, Francis GS, Simon AB, Rector T. Plasma norepinephrine as a guide to prognosis in patients with chronic congestive heart failure. N. Engl. J. Med. 1984; 311:819-823. [PubMed: 6382011]

11. Mani K. Programmed cell death in cardiac myocytes: strategies to maximize post-ischemic salvage. Heart Fail. Rev.. 2008; 13:193-209. [PubMed: 18176842]

12. Ellison GM, Torella D, Karakikes I, Purushothaman S, Curcio A, Gasparri C, Indolfi C, Cable NT, Goldspink DF, Nadal-Ginard B. Acute beta-adrenergic overload produces myocyte damage through calcium leakage from the ryanodine receptor 2 but spares cardiac stem cells. J. Biol. Chem. 2007; 282:11397-11409. [PubMed: 17237229]

13. Wencker D, Chandra M, Nguyen K, Miao W, Garantziotis S, Factor SM, Shirani J, Armstrong RC, Kitsis RN. A mechanistic role for cardiac myocyte apoptosis in heart failure. J. Clin. Invest. 2003; 111:1497-1504. [PubMed: 12750399]

14. Leenen FHH, Skarda V, Yuan B, White R. Changes in cardiac ANG II postmyocardial infarction in rats: effects of nephrectomy and ACE inhibitors. Am. J. Physiol. 1999; 276:H317-H325.

[PubMed: 9887046]

15. Gross PM, Weindl A. Peering through the windows of the brain. J. Cereb. Blood Flow Metab. 1987; 7:663-672. [PubMed: 2891718]

16. Johnson AK, Gross PM. Sensory circumventricular organs and brain homeostatic pathways. FASEB J. 1993; 7:678-686. [PubMed: 8500693]

17. Simpson JB. The circumventricular organs and the central actions of angiotensin. Neuroendocrinology. 1981; 32:248-256. [PubMed: 7012657]

18. Brody, MJ.; Faber, JE.; Mangiapane, ML.; Porter, JP. The central nervous system and prevention of hypertension. In: de Jong, W., editor. Handbook of Hypertension: Experimental and Genetic Models of Hypertension. New York: Elsevier: 1984. p. 474-494.

19. Francis J, Wei SG, Weiss RM, Beltz T, Johnson AK, Felder RB. Forebrain-mediated adaptations to myocardial infarction in the rat. Am. J. Physiol. 2002; 282:H1898-H1906.

20. Felder RB, Francis J, Weiss RM, Zhang Z, Wei SG, Johnson AK. Neurohumoral regulation in ischemia-induced heart failure. Ann. NY Acad. Sci. 2001; 940:444-53. [PubMed: 11458700]

21. Sun Y. Oxidative stress and cardiac repair/remodeling following infarction. Am. J. Med. Sci. 2007; 334:197-205. [PubMed: 17873534]

22. Lindley TE, Doobay MF, Sharma RV, Davisson RL. Superoxide is involved in the central nervous system activation and sympathoexcitation of myocardial infarction-induced heart failure. Circ. Res. 2004; 94:402-409. [PubMed: 14684626]

23. Lindley TE, Infanger DW, Rishniw M, Zhou Y, Doobay MF, Sharma RV, Davisson RL. Scavenging superoxide selectively in mouse forebrain is associated with improved cardiac function and survival following myocardial infarction. Am. J. Physiol. 2009; 296:R1-R8.

24. Infanger DW, A Braga V, Stupinski JA, Sharma RV, Davisson RL. Targeted Knockdown of Nox4 in the Paraventricular Nucleus (PVN) Normalizes Sympathoexcitation and Improves Cardiac Function Following Myocardial Infarction. Hypertension. 2008; 52:e127.

25. Teichholz LE, Kreulen T, Herman MV, Gorlin R. Problems in echocardiographic volume determinations: echocardiographic-angiographic correlations in the presence of absence of asynergy. Am J Cardiol. 1976; 37(1):7-11. [PubMed: 1244736]

26. Zimmerman MC, Lazartigues E, Sharma RV, Davisson RL. Hypertension caused by angiotensin II infusion involves increased superoxide production in the central nervous system. Circ. Res. 2004; 95:210-216. [PubMed: 15192025]

27. Leenen FH. Brain mechanisms contributing to sympathetic hyperactivity and heart failure. Circ. Res. 2007; 101:221-223. [PubMed: 17673679]

28. Watson AM, Hood SG, May CN. Mechanisms of sympathetic activation in heart failure. Clin. Exp. Pharmacol. Physiol. 2006; 33:1269-1274. [PubMed: 17184514]

29. Zucker IH, Wang W, Pliquett RU, Liu JL JL, Patel KP. The regulation of sympathetic outflow in heart failure: the roles of angiotensin II, nitric oxide and exercise training. Ann. N. Y. Acad. Sci. 2001; 940:431-43. [PubMed: 11458699] 
30. Gao L, Wang W, Li YL, Schultz HD, Liu D, Cornish KG, Zucker IH. Superoxide mediates sympathoexcitation in heart failure: roles of angiotensin II and NAD(P)H oxidase. Circ Res. 2004; 95:937-944. [PubMed: 15459075]

31. Gao L, Wang W, Li YL, Schultz HD, Liu D, Cornish KG, Zucker IH. Sympathoexcitation by central ANG II: roles for AT1 receptor upregulation and NAD(P)H oxidase in RVLM. Am. J. Physiol. 2005; 288(5):H2271-H2279.

32. Zhang Z, Francis J, Weiss RM, Felder RB. The renin angiotensin aldosterone system excites hypothalamic paraventricular nucleus neurons in heart failure. Am. J. Physiol. 2002; 283:H423H433.

33. Zucker IH. Novel mechanisms of sympathetic regulation in chronic heart failure. Hypertension. 2006; 48(6):1005-11. [PubMed: 17015773]

34. Zhu GQ, Gao L, Li Y, Patel KP, Zucker IH, Wang W. AT1 receptor mRNA antisense normalizes enhanced cardiac sympathetic afferent reflex in rats with chronic heart failure. Am. J. Physiol. 2004; 287(4):H1828-H1835.

35. Liu JL, Zucker IH. Regulation of sympathetic nerve activity in heart failure: a role for nitric oxide and angiotensin II. Circ. Res. 1999; 84:417-423. [PubMed: 10066676]

36. Wang H, Huang BS, Ganten D, Leenen FHH. Prevention of sympathetic and cardiac dysfunction after myocardial infarction in transgenic rats deficient in brain angiotensinogen. Circ. Res. 2004; 94:843-849. [PubMed: 15061159]

37. Lal A, Veinot JP, Ganten D, Leenen FHH. Prevention of cardiac remodeling after myocardial infarction in transgenic rats deficient in brain angiotensinogen. J. Mol. Cell. Cardiol. 2005; 39:521-529. [PubMed: 15950985]

38. Zanzinger J, Czachurski J. Chronic oxidative stress in the RVLM modulates sympathetic control of circulation in pigs. Pflugers Arch. 2000; 439:489-494. [PubMed: 10678747]

39. Mani K. Programmed cell death in cardiac myocytes: strategies to maximize post-ischemic salvage. Heart Fail. Rev. 2008; 13:193-209. [PubMed: 18176842]

40. Sun Y. Oxidative stress and cardiac repair/remodeling following infarction. Am. J. Med. Sci. 2007; 334:197-205. [PubMed: 17873534] 

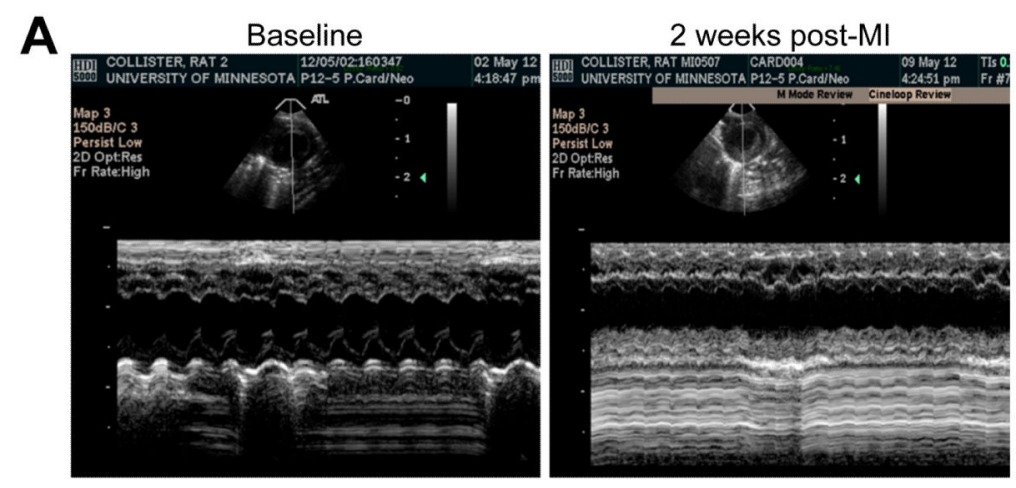

B

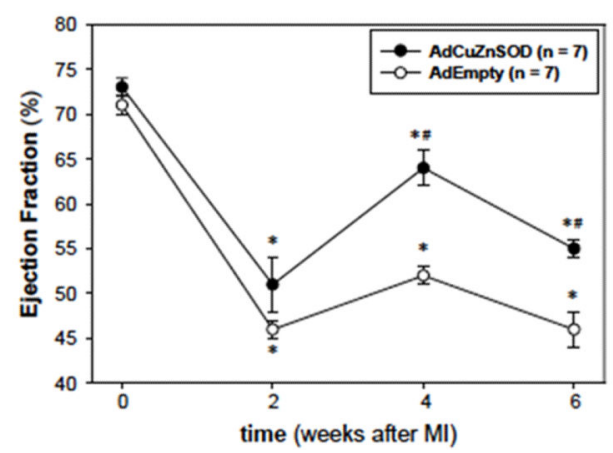

Figure 1.

A) Representative M-mode echocardiograms from a rat at baseline and a rat 2 weeks postMI showing normal left ventricular chamber dimensions and normal systolic function at baseline versus increased size of the left ventricular chamber dimensions and markedly depressed left ventricular systolic function at 2 weeks post-MI. B) Summary data of ejection fractions (EF) from rats injected with AdCuZnSOD or AdEmpty into the MnPO demonstrating a significant decrease in EF in both groups at 2 weeks post-MI. EF was diminished less in AdCuZnSOD rats at 4 and 6 weeks post MI compared to AdEmpty rats (* $=\mathrm{p}<0.05$ compared to baseline, $\#=\mathrm{p}<0.05$ compared to AdEmpty at the respective time point). 


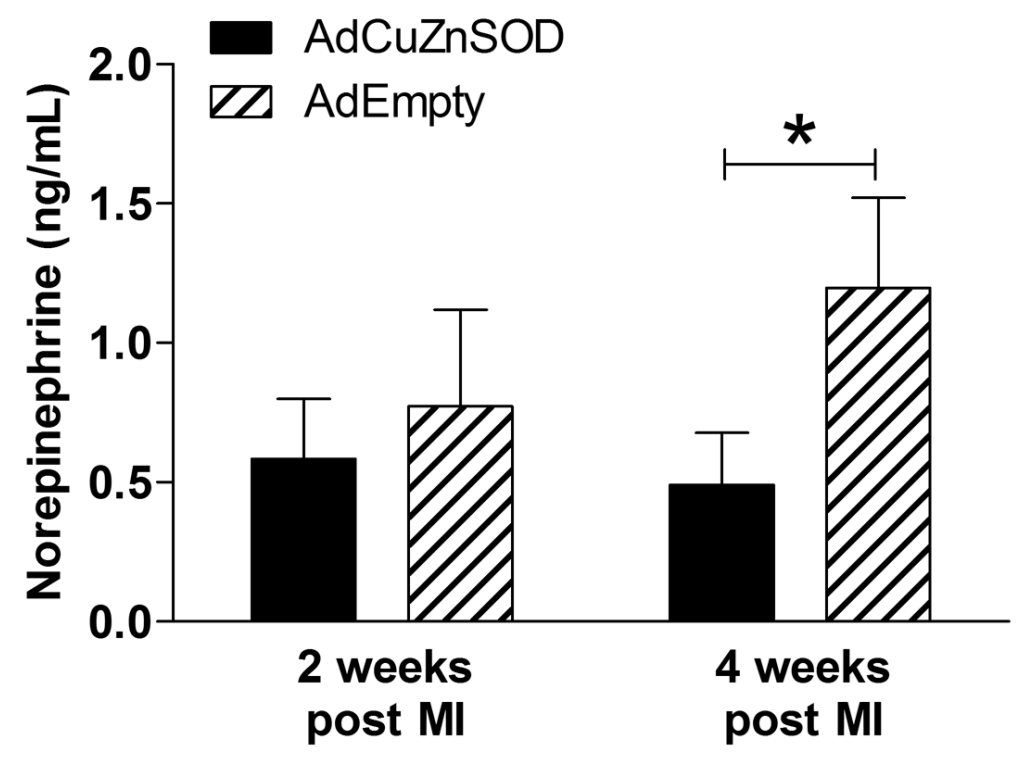

Figure 2.

Summary data of plasma norepinephrine levels from AdCuZnSOD- and AdEmpty-injected rats demonstrating similar plasma norepinephrine levels in both groups at 2 weeks post MI, and a significant increase in plasma norepinephrine in AdEmpty treated rats at 4 weeks post MI. $(*=p<0.05$ between groups $)$. 


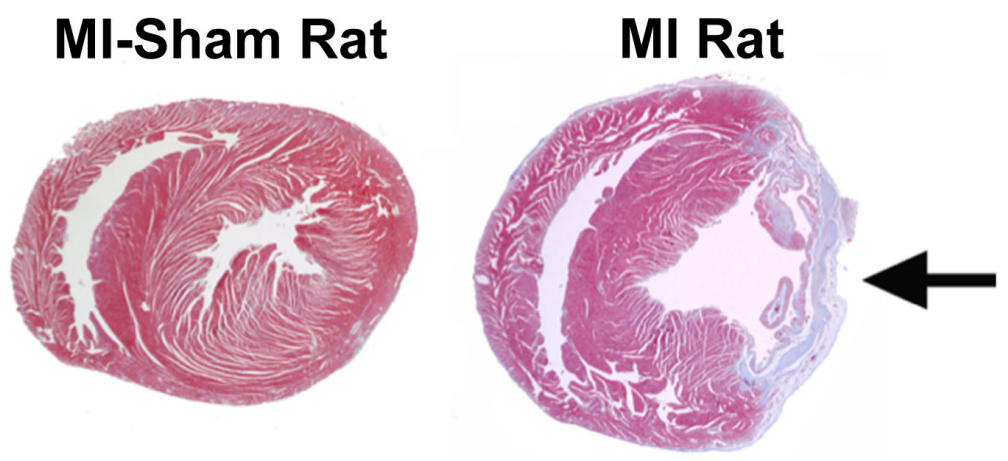

Figure 3.

Representative transverse histological sections of the mid-ventricle from hearts of a MISham rat (left image) and a MI rat (right image). Both normal right and left ventricles are shown in the MI-Sham rat compared to a highly infarcted area (ARROW) of the free wall of the left ventricle a MI rat. Sections are stained with Masson Trichrome (blue staining = collagen) highlighting the loss of myocardium and replacement with fibrous connective tissue in the MI rat. 

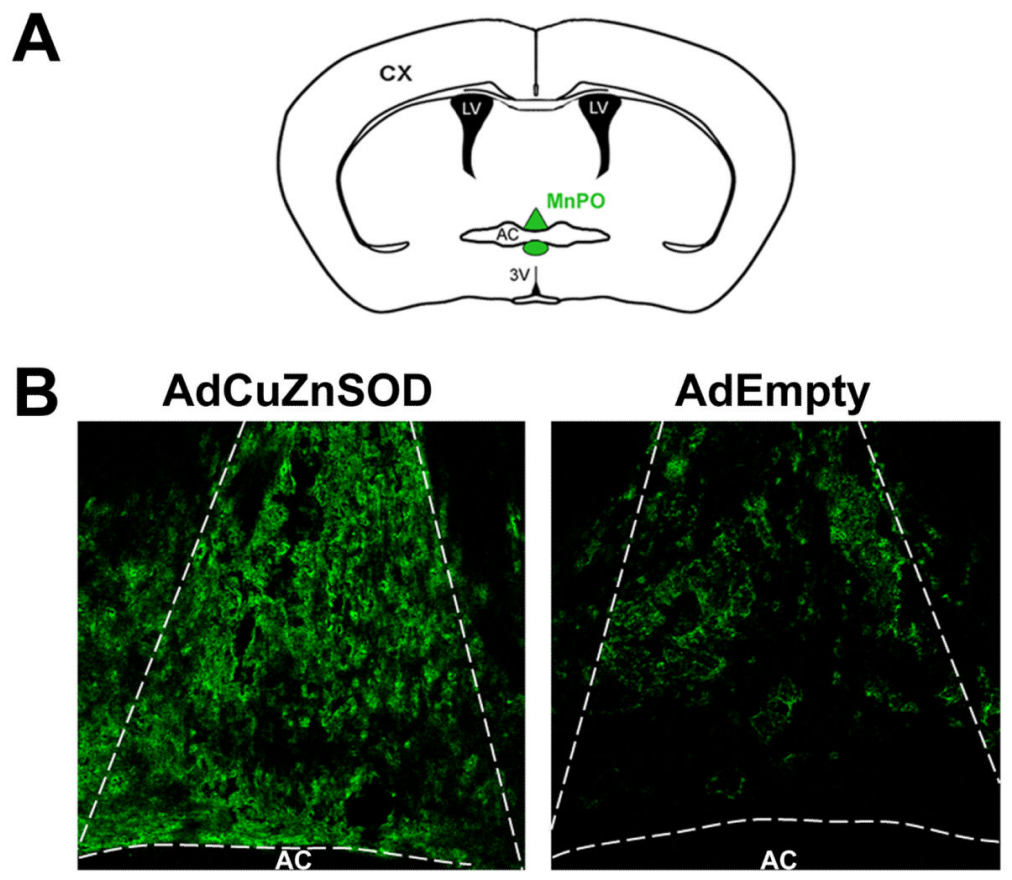

Figure 4.

A) Schematic of a coronal forebrain section showing the location of the MnPO (highlighted in green). $\mathrm{CX}$ - cortex; $\mathrm{LV}$ - lateral ventricle; $\mathrm{AC}$ - anterior commissure; $3 \mathrm{~V}$ - third ventricle). B) Immunofluorescent staining of CuZnSOD in the MnPO 6 weeks after direct $\mathrm{MnPO}$ injection of AdCuZnSOD or AdEmpty demonstrating a marked increase in CuZnSOD protein levels (green fluorescence) in AdCuZnSOD rats compared to AdEmpty rats. White dotted lines indicate border of dorsal MnPO, which lies just dorsal to the anterior commissure (AC). 
Table 1

Summary of ejection fraction data pre- and post-MI between AdCuZnSOD and AdEmpty rats.

\begin{tabular}{|c|c|c|c|c|}
\hline Ejection Fraction (\%) & Baseline & 2 week post MI & 4 weeks post MI & 6 weeks post MI \\
\hline AdCuZnSOD $(n=7)$ & $73 \pm 1$ & $51 \pm 3 *$ & $64 \pm 2^{* \dagger}$ & $55 \pm 1^{*} \dagger$ \\
\hline AdEmpty $(n=7)$ & $71 \pm 1$ & $46 \pm 1^{*}$ & $52 \pm 1$ * & $46 \pm 2 *$ \\
\hline
\end{tabular}

